\title{
Syphilis reinfections pose problems for syphilis diagnosis in Antwerp, Belgium - 1992 to 2012
}

C Kenyon (ckenyon@itg.be) ${ }^{1}$, L Lynen ${ }^{1}$, E Florence ${ }^{1}$, S Caluwaerts $^{1}$, M Vandenbruaene ${ }^{1}$, L Apers ${ }^{1}$, P Soentjens ${ }^{1}$, M Van Esbroeck ${ }^{1}$, E Bottieau ${ }^{1}$

1. Institute for Tropical Medicine, Antwerp, Belgium

Citation style for this article:

Kenyon C, Lynen L, Florence E, Caluwaerts S, Vandenbruaene M, Apers L, Soentjens P, Van Esbroeck M, Bottieau E. Syphilis reinfections pose problems for syphilis diagnosis in Antwerp, Belgium - 1992 to 2012. Euro Surveill. 2014;19(45):pii=20958. Available online: http://www.eurosurveillance.org/ViewArticle. aspx?Articleld $=20958$

Article submitted on 30 October 2013 / published on 13 November 2014

Persons with multiple syphilis reinfections may play an important role in syphilis transmission. We analysed all syphilis tests carried out for people attending the HIV/sexually transmitted infection (STI) clinic at the Institute of Tropical Medicine, Antwerp, Belgium, from 1992 to 2012 to evaluate the extent to which syphilis reinfections were contributing to the syphilis epidemic in Antwerp. We then characterised the features of the syphilis infections in individuals with five or more episodes of syphilis. A total of 729 syphilis episodes were diagnosed in 454 persons. The majority of syphilis episodes occurred in people who had more than one episode of syphilis (445/729; $61 \%$ ). A total of 10 individuals had five or more episodes of syphilis diagnosed over this period. All were men who have sex with men, HIV positive and on antiretroviral therapy. They had a total of 52 episodes of syphilis diagnosed and treated. In 38/42 of the episodes of repeat syphilis in these 10 individuals, they presented without any signs or symptoms of syphilis. Given that the majority of cases of incident syphilis in our clinic were persons with reinfections and that they frequently presented without signs of symptoms of syphilis, there is a strong case for frequent and repeated screening in all persons with a diagnosis of syphilis.

\section{Introduction}

Syphilis is a sexually transmitted infection (STI) caused by Treponema pallidum subspecies pallidum. Its protean clinical manifestations depend on the stage of disease [1]. If a person presents in the primary stage, this typically presents as a painless chancre - after an incubation period of 10-90 days. In the secondary stage, there is a diffuse rash and in tertiary syphilis, there are neurological, cardiovascular or gummatous lesions. There are no symptoms in the latent phase, which occurs between the primary/secondary and tertiary phases [1]. Since 2000 , there has been a considerable increase in the incidence of syphilis in a number of high-income countries, such as Belgium, Czech Republic, Denmark, Greece, Luxembourg, the Netherlands, Norway, Slovakia, Slovenia and Spain [2]. These outbreaks have occurred predominantly in men who have sex with men (MSM), many of whom are HIV positive [2-4]. In Belgium, the incidence of syphilis was 12 cases per 100,000 population in 2012 [5].

A number of studies have pointed out the importance of core groups in the genesis of the current syphilis outbreaks in high-income countries [3,4,6-8]. In some studies, it has been argued that persons with multiple syphilis reinfections are more likely to have high numbers of sexual partners and to be involved in highrisk sexual networks. As a result they may constitute a core-within-the-core and play an especially important role in syphilis spread $[8,9]$.

In addition, there is recent evidence that syphilis is frequently diagnosed concomitantly with hepatitis C (HCV) infection in HIV-positive MSM and might play a role in HCV transmission [5]. The current syphilis epidemic in Antwerp, which started around 2000, has been characterised by a large number of clients who have repeated episodes of syphilis (repeat syphilis) $[10,11]$.

The diagnosis of $T$. pallidum reinfection may be more difficult than the diagnosis of the first episode of syphilis. There are three possible reasons for this. Firstly, it is difficult to distinguish syphilis reinfection from disease relapse, since the diagnosis of both of these depends on clinical findings of syphilis and a fourfold increase in non-treponemal test titres $[12,13]$. Secondly, syphilis infection leads to partial immunity to reinfection and thus subsequent episodes of syphilis may not present in the same way as initial episodes $[9,14,15]$. Thirdly, in settings where syphilis is regularly screened for in high-risk persons, syphilis is more likely to be diagnosed earlier, before the development of clinical symptoms $[7,16,17]$. These last two factors mean that a large proportion of repeat syphilis may be diagnosed purely on the basis of changes in titres of non-treponemal tests. Both the sensitivity and the specificity of these tests are suboptimal at various stages of syphilis [18]. As a result, the diagnosis of repeat syphilis may be both under- and over-diagnosed. 
Given these diagnostic difficulties, we undertook a study of syphilis reinfections in persons attending the Institute of Tropical Medicine's (ITM) HIV/STI clinic in Antwerp, Belgium, with the following aims: to establish the proportion of syphilis episodes occurring in persons with a previous diagnosis of syphilis; to characterise the features of the syphilis infections in persons with five or more episodes of syphilis; and to discuss the optimal diagnostic and screening strategies in individuals with repeat syphilis.

\section{Methods}

We conducted a laboratory review of all syphilis tests carried out for persons attending the HIV/STI clinic at the ITM from 1 January 1992 to 31 December 2012. From 2000, people at-risk attending the clinic were routinely screened 6-12 monthly with a rapid plasma reagin (RPR) test (Becton, Dickinson and Company, United States (US)) - a non-treponemal test - and a $T$. pallidum particle agglutination (TPPA) test (Fujirebio Inc., Japan). Persons at-risk were defined as those with high-risk sexual behaviour or a history of a previous diagnosis of an STI.

An episode of syphilis was defined as an episode in which a person who had an RPR titre $\geq 1 / 8$ and $a \geq 4$-fold increase in RPR titres from a previous RPR titre and a positive TPPA test on serum. Previous RPR results were not always available and thus the definition also applied to persons who on their first visit had a positive TPPA and an RPR titre $\geq 1 / 8$ and the RPR titre fell $\geq 4$-fold following appropriate therapy. A repeat episode of syphilis, which included syphilis reinfections and reactivations, was defined as an episode in a person who had a $\geq 4$-fold increase in RPR titre, after a previous diagnosis of syphilis who exhibited an appropriate response to therapy ( $\geq 4$-fold decrease in RPR). If there were signs of primary, secondary, tertiary or neurosyphilis at the time of the repeat syphilis, then the fourfold decrease was not required.

Detailed folder reviews were carried out for all persons who had had five or more episodes of syphilis recorded over the study period. We focused on clients with five or more episodes of syphilis because we considered that these individuals may represent persons in a core sexual network that could be particularly important for syphilis transmission. Data extracted for this subgroup included demographics, clinical details including other STI diagnoses made between 1992 and 2012 and the clinical stage of syphilis at the time of diagnosis and the therapy administered.

Stages of syphilis were determined by an infectious disease specialist according to US Centers for Disease Control and Prevention surveillance definitions $[19,20]$. Latent syphilis was defined as syphilis characterised by RPR/TPPA seroreactivity without other evidence of disease. Persons who had latent syphilis and who acquired syphilis during the preceding year were classified as having early latent syphilis. Men who have
TABLE

Syphilis diagnoses stratified by number of episodes of syphilis per person attending Institute of Tropical Medicine's HIV/sexually transmitted infections clinic, Antwerp, Belgium, 1992-2012 $(\mathrm{n}=454)$

\begin{tabular}{|l|c|c|}
\hline $\begin{array}{l}\text { Number of episodes } \\
\text { of syphilis (A) }\end{array}$ & $\begin{array}{c}\text { Number of persons } \\
\text { (B) }\end{array}$ & $\begin{array}{c}\text { Total number of } \\
\text { episodes of syphilis } \\
(\text { A } \times \text { B) }\end{array}$ \\
\hline 1 & 284 & 284 \\
\hline 2 & 100 & 200 \\
\hline 3 & 47 & 141 \\
\hline 4 & 13 & 52 \\
\hline 5 & 8 & 40 \\
\hline 6 & 2 & 12 \\
\hline Total & 454 & 729 \\
\hline
\end{tabular}

sex with men (MSM) were defined as men who reported ever having had sex with men.

The data for the number of syphilis cases diagnosed in the same time period for the Province of Antwerp were obtained from the Belgian Scientific Institute of Public Health. Ethical approval for the study was obtained from the Institutional Review Board of the ITM.

\section{Statistics}

The chi-squared test for trend was used to assess if repeat episodes of syphilis represented an increasing proportion of the total number of syphilis cases per year. All analyses were conducted in Stata 12.0 (Stata Corp, College Station, TX, US).

\section{Results}

A total of 3,581 individuals were tested for syphilis between 1992 and 2012 in the ITM HIV/STI clinic. Some 729 episodes of syphilis were diagnosed in 454 persons, including 284 considered as first episodes and 445 as repeat syphilis (Table).

Table. Syphilis diagnoses stratified by number of epi The syphilis episodes observed at the ITM clinic constituted $44 \%(729 / 1,662)$ of all episodes of syphilis diagnosed in the Province of Antwerp over the study period. Syphilis was only diagnosed in persons who were HIV positive at the time of the diagnosis or who became HIV positive at a later date. There were no cases of syphilis diagnosed from 1992 to 1994; most syphilis diagnoses occurred after 2000 (Figure 1). The majority of episodes of syphilis occurred in people who had more than one episode of syphilis $(445 / 729 ; 61 \%)$ (Table). The proportion of infections due to repeat syphilis increased over the study period $(p<0.001)$ (Figure 1$)$.

\section{Clients with five episodes of syphilis}

A total of 10 persons had five or more episodes of syphilis diagnosed over the study period (Figure 2). All were MSM, HIV positive and on antiretroviral therapy 
Cases of initial and repeat syphilis diagnosed per year at the Institute of Tropical Medicine, Antwerp, Belgium, $1992^{\mathrm{a}}-2012(\mathrm{n}=729)$

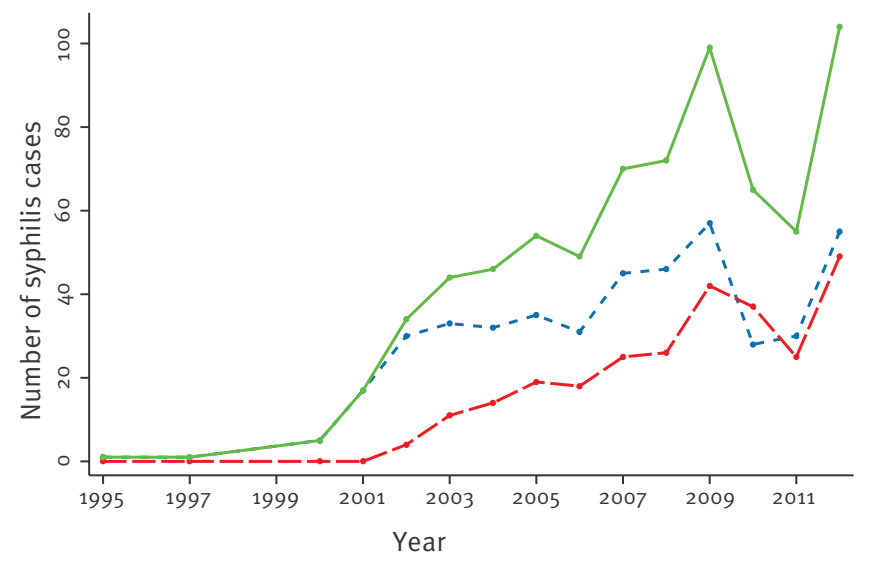

- - - - First episode of syphilis

$\longrightarrow$ Total number of syphilis cases

$\rightarrow-$ Repeat episode of syphilis

The graph begins in 1995 as there were no cases of syphilis diagnosed during 1992 to 1994.

with good virological and immunological responses. They had a total of 52 episodes of syphilis diagnosed and treated. The clinical stage of syphilis could be determined in all 10 first episodes of syphilis, but in only four of the 42 repeat episodes (Figure 2). All the remaining 38 repeat episodes were picked up by screening tests.

The 10 clients were diagnosed with one to eight (median: two) other STIs over the study period (Figure 2). This excluded HIV and hepatitis B, for which all 10 demonstrated evidence of previous or current infection.

The RPR titres declined by the requisite fourfold amount within a year following appropriate therapy in each of the diagnoses of syphilis, excluding the third episode in Case 9, where it took two years for this drop to occur.

The RPR/TPPA tests (both of which are routinely performed on the same serum specimen in our laboratory) were done a mean of 152 days apart (95\% Cl: $33-387$ days).

\section{Discussion}

The incidence of syphilis continues to increase in a number of European countries [2-4,21-23] In many of these countries, the majority of these infections are occurring in MSM [2,3,23]. In our study, individuals with repeat syphilis infections constituted an increasing proportion of all syphilis infections in people visiting our clinic in Antwerp. They now constitute the majority of syphilis infections seen in our clinic.
As early as 1926, it was suggested that an increase in titre of one of the non-treponemal tests without symptoms or signs of syphilis could represent reinfection [21]. Great debate has followed about the relative importance of reinfection versus relapse in the aetiology of symptomless syphilis $[24,25]$ and whether or not repeat syphilis presents in the same way as initial syphilis $[9,25]$.

In the current outbreaks of syphilis in high-income countries in Europe and elsewhere, retrospective analyses of syphilis diagnoses within specific geographical areas have reached different conclusions as whether or not there are differences in how initial and repeat episodes of syphilis present. A study of MSM from San Diego, California, US, in 2004-07 found no difference [26], whereas other US studies from Florida and Seattle, Washington, in 2000-08 and 1992-2008, respectively, found that persons with repeat syphilis were more likely to present with asymptomatic disease - although in the Florida study this was only statistically significant for the HIV-infected MSM group $[9,27]$. In the Seattle study, repeat syphilis presented as early latent disease in $113 / 254(44.5 \%)$ cases, while $309 / 1,191$ (25.9\%) cases with initial syphilis [9].

A consistent finding in studies in which individuals in a high-risk group are regularly screened for syphilis is that a relatively high proportion (with both initial onset and repeat syphilis) are found to have asymptomatic disease of recent onset. In a study that involved threemonthly RPR and TPPA screening of an HIV-positive cohort in London, England, in 2002, for example, most (26/44) new diagnoses of early syphilis were asymptomatic [15]. A study from the Netherlands in 2003 using comparable methodology had similar findings [3].

\section{Why are repeat episodes so often asymptomatic in HIV-positive MSM?}

There could be at least five explanations for the high proportion of persons identified with asymptomatic syphilis in the setting of syphilis screening programmes.

(i) False-positive test results.

There is a long list of causes of increases in the titres of the non-treponemal tests that are unrelated to syphilis $[1,18]$. Frequent testing may be more likely to detect these increases and misdiagnose them as asymptomatic syphilis.

(ii) Syphilis relapse.

Ineffective treatment may lead to relapse [17].

\section{(iii) Lead-time bias.}

The regular sampling (up to three monthly) involved in the screening programmes would make it more likely that persons are diagnosed during the syphilis incubation period before they develop symptoms of syphilis $[7,16,17]$. 
Changes in test ${ }^{\text {a }}$ titres and diagnoses of new sexually transmitted infections in 10 cases with five or more episodes of syphilis diagnosed between 1992 and 2012, Institute of Tropical Medicine, Antwerp, Belgium
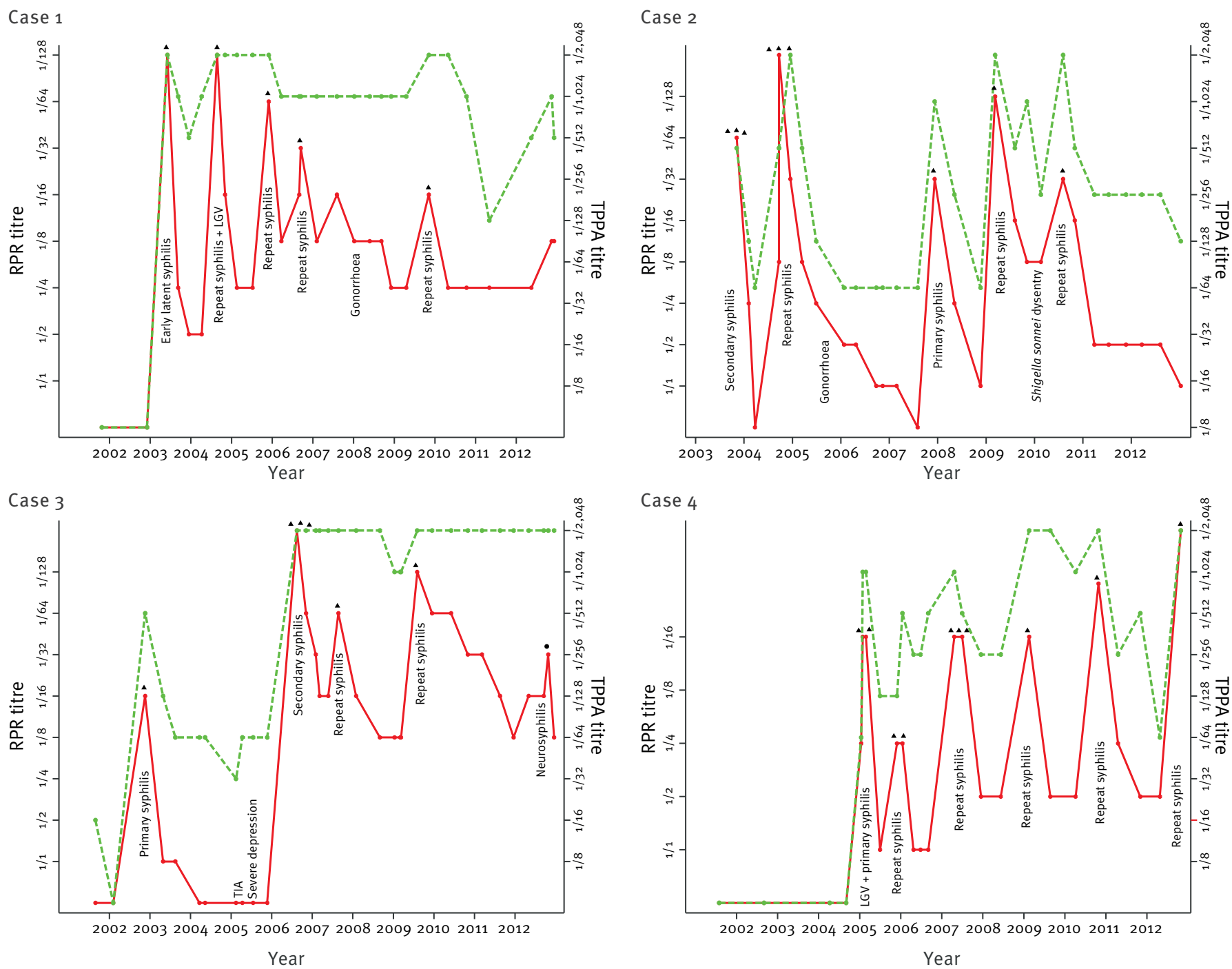

Year

- Benzathine penicillin intramuscular injection

- 24 million units penicillin $\mathrm{G}$ therapy/day intravenous for 10 days

$\rightarrow$ RPR

- Doxycycline 10omg twice a day for 21 days

CT: Chlamydia trachomatis; HAV: hepatitis A virus; HCV: hepatitis C virus; LGV: lymphogranuloma venereum; RPR: rapid plasma regain; TIA: transient ischaemic attack; TPPA: Treponema pallidum particle agglutination.

a RPR and TPPA tests.

The denominators of all TPPA titres were divided by 10 due to space constraints.

(iv) Partial immunity.

Screening programmes have been taking place in high-risk populations, in which an increasing proportion of syphilis is due to reinfection $[15,26]$. T. pallidum infection induces partial immunity to reinfection in both humans and other animals, which may lead to an increased proportion of repeat infections being asymptomatic $[14,15,24]$.

(v) Missed signs of disease.

Clinicians in screening programmes may place undue reliance on serological tests at the expense of clinical evaluations and thus be more likely to miss clinical signs of disease.

Although false-positive RPR tests and relapses can occur, we do not believe that these are dominant factors in why repeat episodes of syphilis are so often asymptomatic. The most likely causes of false-positive RPR tests in our study population, such as vaccinations and other infections $[1,28]$ tend to cause small increases in RPR titres - not the large increases that occurred in all of the episodes seen here. Furthermore, they would not lead to the increases in TPPA titres that characterise reinfections. Since all the cases received 
Changes in test ${ }^{\mathrm{a}}$ titres and diagnoses of new sexually transmitted infections in 10 cases with five or more episodes of syphilis diagnosed between 1992 and 2012, Institute of Tropical Medicine, Antwerp, Belgium
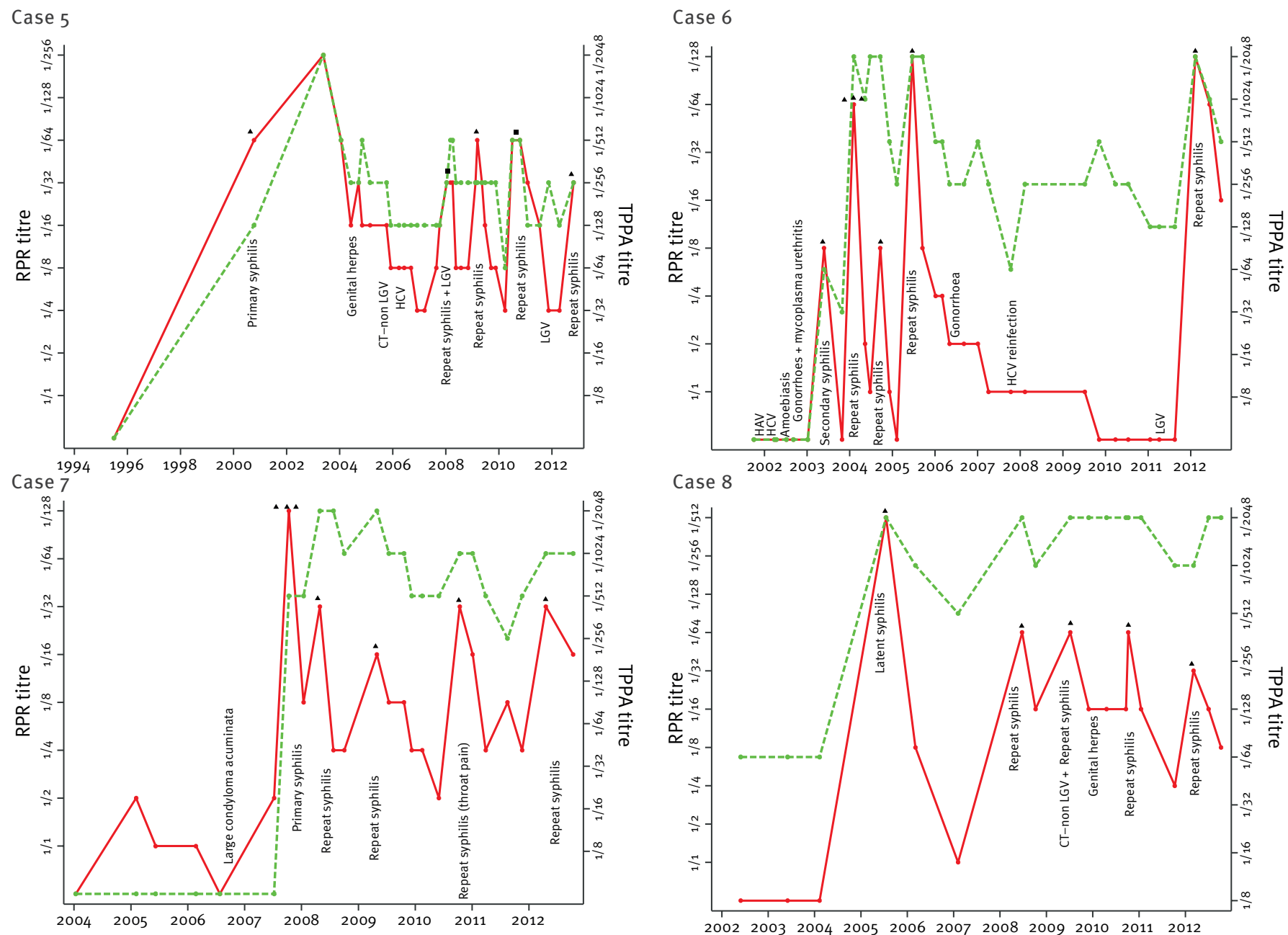

Case 8

20022003200420052006200720082009201020112012

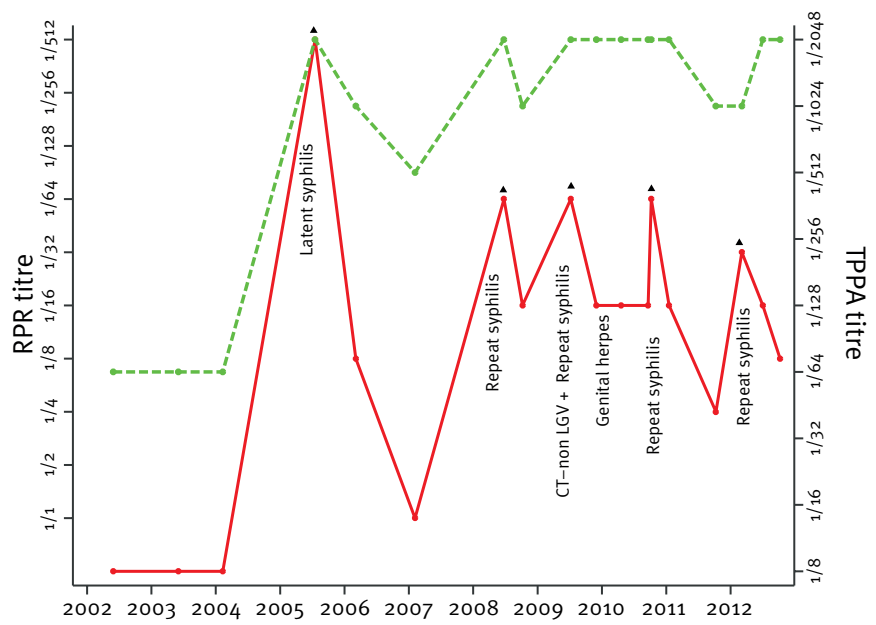

$\begin{array}{lll}\rightarrow & \text { RPR } & \text { Benzathine penicillin intramuscular injection } \\ \rightarrow-- & \text { TPPA } & 24 \text { million units penicillin } \mathrm{G} \text { therapy/day intravenous for } 10 \text { days }\end{array}$

CT: Chlamydia trachomatis; HAV: hepatitis A virus; HCV: hepatitis C virus; LGV: lymphogranuloma venereum; RPR: rapid plasma regain; TIA: transient ischaemic attack; TPPA: Treponema pallidum particle agglutination.

a RPR and TPPA tests.

The denominators of all TPPA titres were divided by 10 due to space constraints.

stage-appropriate, highly effective therapy under direct supervision that has an extremely low failure rate [29], we assume that relapses were very unlikely.

The lead-time bias explanation likely explains only a proportion of asymptomatic presentations. This can be inferred from comparing the incubation period of syphilis, being a mean of 21 days (range: $10-90$ days) $[1,12]$ and the time to positivity of an RPR test (ca $50 \%$ and $90 \%$ positive by three and six weeks post infection, respectively) [29] with the frequency of RPR testing (median: 114 days; $3 \%, 7 \%$ and $28 \%$ of tests were performed at 7,14 and 90 days post infection, respectively). As only $28 \%$ of tests were performed within the maximum 90-day incubation period, this suggests that the lead-time bias is unable to explain more than a minority of the $91 \%$ of the repeat cases presenting asymptomatically.

Regarding clinicians missing signs of disease, we do not consider this as a likely explanation as this would not explain why only $2 / 10$ of the initial episodes of syphilis (vs 39/42 cases of repeat syphilis) were diagnosed as asymptomatic syphilis. 
Changes in test ${ }^{\text {a }}$ titres and diagnoses of new sexually transmitted infections in 10 cases with five or more episodes of syphilis diagnosed between 1992 and 2012, Institute of Tropical Medicine, Antwerp, Belgium
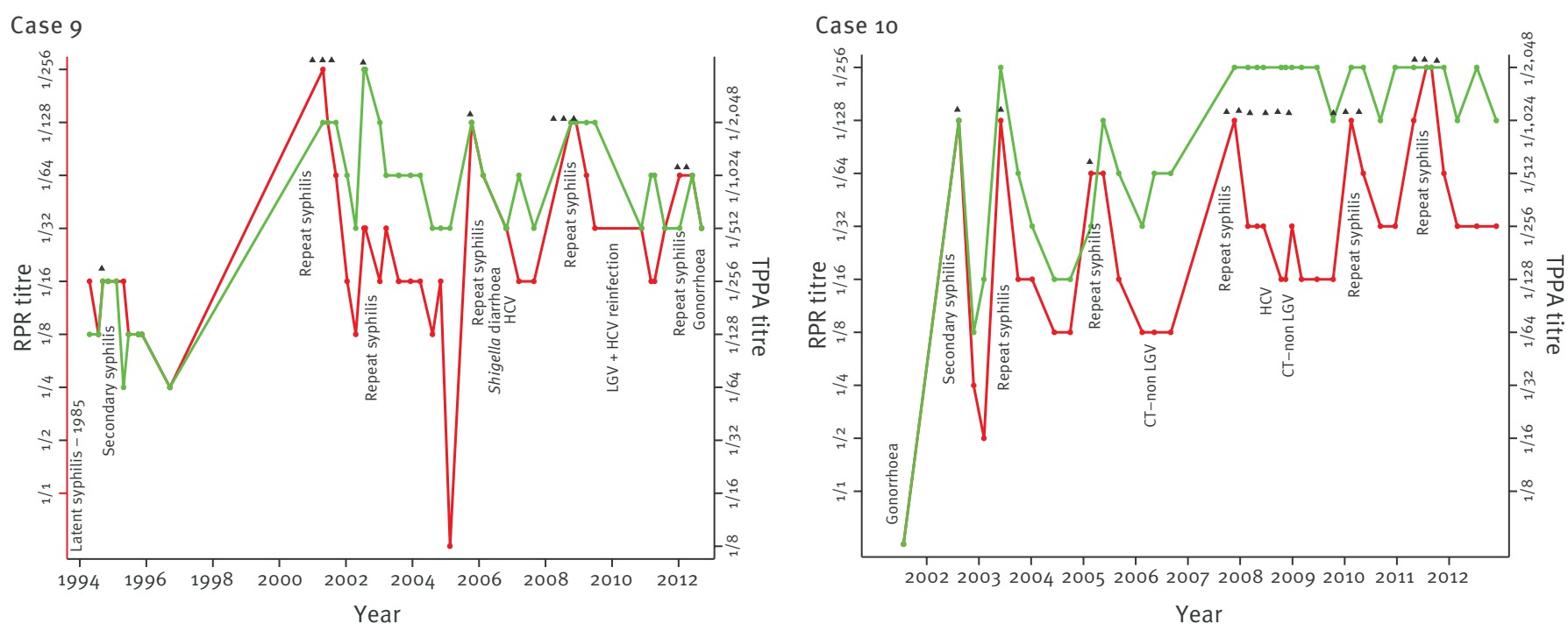

$\begin{array}{lll}\rightarrow \text { RPR } & \text { B } & \text { Benzathine penicillin intramuscular injection } \\ -\infty=- \text { TPPA } & \text { - } 24 \text { million units penicillin } \mathrm{G} \text { therapy/day intravenous for } 10 \text { days }\end{array}$

CT: Chlamydia trachomatis; HAV: hepatitis A virus; HCV: hepatitis C virus; LGV: lymphogranuloma venereum; RPR: rapid plasma regain; TIA: transient ischaemic attack; TPPA: Treponema pallidum particle agglutination.

a RPR and TPPA tests.

The denominators of all TPPA titres were divided by 10 due to space constraints.

These considerations suggest that lead-time bias and partial immunity may both play a role in the way that repeat syphilis presents asymptomatically. Although our findings are based on small numbers, may not be generalisable to other populations undergoing screening and require replication, they have important implications. They suggest that persons with multiple episodes of syphilis may be more likely to present with asymptomatic disease. Because untreated syphilis may remain infectious for more than a year [29], this could result in these individuals having an even larger role in syphilis transmission than their central position in high-risk sexual networks would have led to. This makes a good case for screening these individuals frequently on a long-term basis. Although further study is required, it may be prudent to screen all individuals with a repeat syphilis diagnosis three to six monthly or, failing that, screen them six monthly with a more sensitive test such as an IgM test. A study from 1999 to 2008 in Zurich, Switzerland, found that an IgM enzymelinked immunosorbent assay was negative in considerably fewer cases of primary syphilis cases compared with the RPR test ( $4 \%$ vs $42 \%$ ) [30].

It should be noted that persons with asymptomatic syphilis are usually defined as having latent syphilis and there is good evidence to suggest that this category of syphilis is less infectious [28]. There are, however, a number of reasons why it is inappropriate to classify the asymptomatic syphilis diagnosed in our cases as latent syphilis. Latency in a number of articles and textbooks is conceived as being the stage following primary/secondary syphilis $[1,12,31]$. In the individuals with reinfections analysed in our study, however, syphilis was either diagnosed before the primary/secondary stages could manifest (during the incubation period) or it presented without signs of primary/secondary disease (true asymptomatic). Thus, we propose that latency be reconceptualised from being a category that follows primary /secondary disease to one which, in addition, can occur before or contemporaneous to primary/secondary disease (Figure 3). Lead-time bias will also mean that some persons will be diagnosed so early that it is not possible to predict what stage they would have presented with if they had not been treated. Further research is necessary to ascertain how infectious individuals with repeat episodes of syphilis are.

More attention needs to be directed to this emerging issue of repeat syphilis. Not only may it play an important role in syphilis transmission but individuals with repeat syphilis may also merit a specific, still to be defined, clinical work-up. This could include a lumbar puncture to exclude relapse from infection arising from a neurological reservoir of $T$. pallidium and additional confirmatory tests (including TPPA tests). The transient ischaemic attack and severe depression that Case 3 


\section{FIGURE 3}

Adjusted conceptual framework of the natural history of untreated syphilis

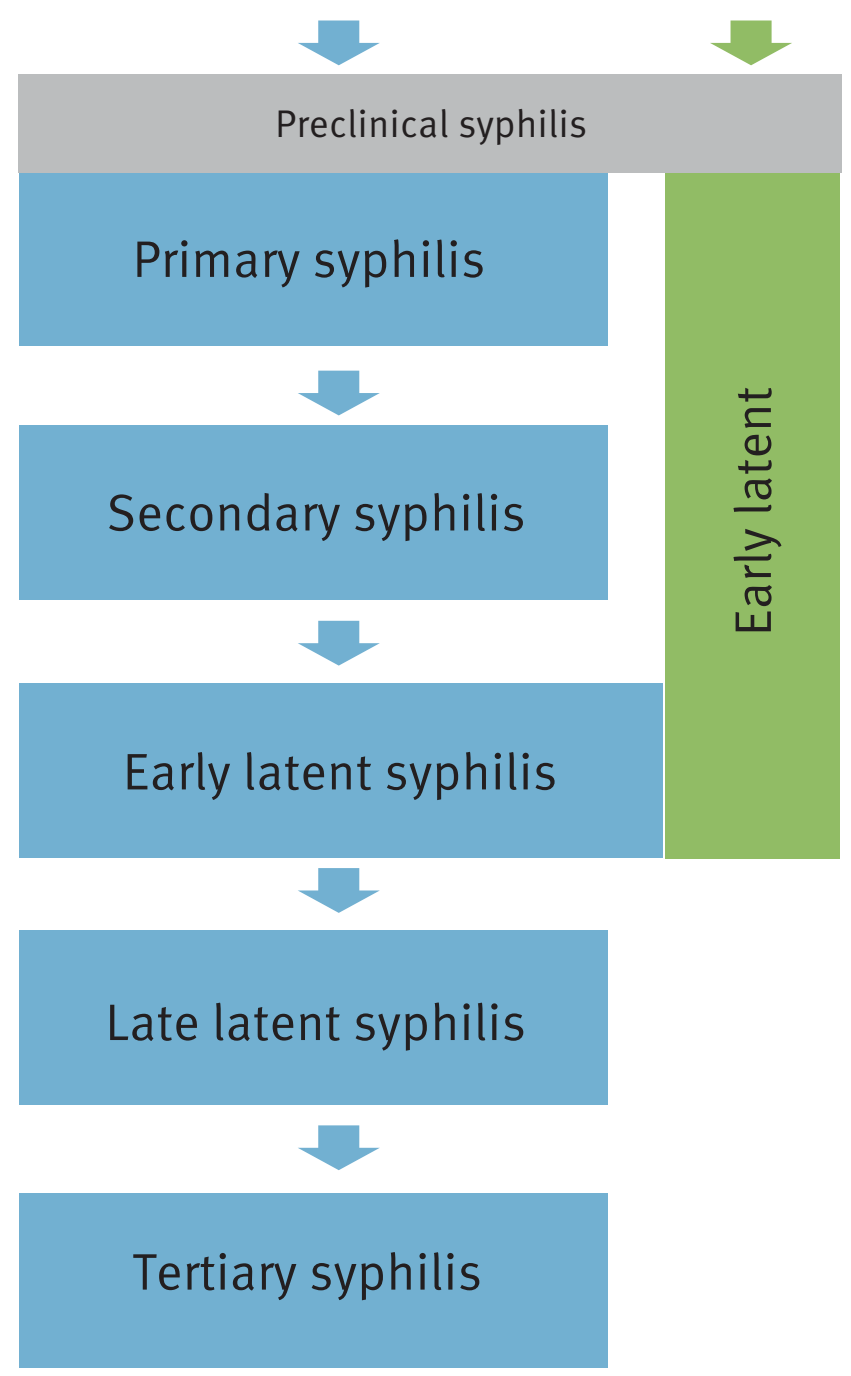

In addition to the traditional framework (depicted in blue), syphilis can be diagnosed at a preclinical stage in the setting of screening (grey) or directly as an early latent stage without having passed through the primary/secondary stages (green). A higher proportion of clients with repeat syphilis may present directly as early latent syphilis.

was diagnosed with between numerous episodes of syphilis may reflect such a neurosyphilis (Figure 2). Although speculative, lumbar puncture at this stage may have led to his neurosyphilis being treated earlier and may have prevented further relapses of syphilis. Optimal therapy for repeat syphilis has also not been completely defined thus far. Finally, interventions that are able to address the risky behaviours underpinning repeat syphilis infections in HIV-positive MSM are urgently required.
Acknowledgements

We would like to thank Ruth Verbrugge from the Belgian Scientific Institute of Public Health for providing the data on the number of syphilis cases for the Province of Antwerp.

\section{Conflicts of interest}

None declared.

\section{Authors' contributions}

Conceived and coordinated the study: Chris Kenyon. Carried out the data analyses and folder reviews: Chris Kenyon. Drafted the manuscript: Chris Kenyon, Emmanuel Bottieau. Contributed to the manuscript: Lut Lynen, Eric Florence, Marc Vandenbruaene, Severine Caluwaerts, Ludwig Apers, Patrick Soentjens, Marjan Van Esbroeck.

\section{References}

1. Tramont E. Syphilis In: Mandell GL, Bennett JE, Dolin R. Principles and practice of infectious diseases. 7 th ed. New York, NY: Churchill Livingstone Inc.; 2010. p. 3035-55.

2. European Centre for Disease Prevention and Control (ECDC). Sexually transmitted infections in Europe 2011. Stockholm: ECDC; 2013. Available from: http://www.ecdc.europa.eu/en/ publications/publications/sexually-transmitted-infectionseurope-2011.pdf

3. Branger J, van der Meer JT, van Ketel RJ, Jurriaans S, Prins JM. High incidence of asymptomatic syphilis in HIV-infected MSM justifies routine screening. Sex Transm Dis. 2009;36(2):845. http://dx.doi.org/10.1097/OLQ.ob013e318186debb PMID:18971797

4. Jakopanec I, Grjibovski AM, Nilsen $\emptyset$, Aavitsland P. Syphilis epidemiology in Norway, 1992-2008: resurgence among men who have sex with men. BMC Infect Dis. 2010;10(1):105. http:// dx.doi.org/10.1186/1471-2334-10-105 PMID:20429881

5. Verbrugge R, Crucitti T, Quoilin S. Surveillance of Sexually Transmitted Infections in the general population of Belgium and its regions. Brussels: Scientific Institute of Public Health; 2013. Available from: https://www.aml-lab.be/website.nsf/ Text/SOA-2012-NL/\$file/SOA-2012-NL.pdf

6. Bellis MA, Cook P, Clark P, Syed Q, Hoskins A. Re-emerging syphilis in gay men: a case-control study of behavioural risk factors and HIV status. J Epidemiol Community Health. 2002;56(3):235-6. http://dx.doi.org/10.1136/jech.56.3.235 PMID:11854349

7. Cohen CE, Winston A, Asboe D, Boag F, Mandalia S, Azadian $B$, et al. Increasing detection of asymptomatic syphilis in HIV patients. Sex Transm Infect. 2005;81(3):217-9. http://dx.doi. org/10.1136/sti.2004.012187 PMID:15923288

8. Fenton KA, Wasserheit JN. The courage to learn from our failures: syphilis control in men who have sex with men. Sex Transm Dis. 2007;34(3):162-5. http://dx.doi.org/10.1097/01. olq.0000259398.70789.c6 PMID:17325602

9. Kerani R, Lukehart S, Stenger M, Marra C, Pedersen R, Golden $M R$. Is early latent syphilis more likely in patients with a prior syphilis infection? Presentation at: 18th International Society for STD Research, London, 28 June-1 July 2009. Available from: http://www.isstdr.org/BASSH $\% 20$ Abstracts $\% 20$ as $\% 20$ at $\% 20$ $16 \% 20$ November.pdf</eref>

10. Sasse A, Defraye A, Ducoffre G. Recent syphilis trends in Belgium and enhancement of STI surveillance systems. Euro Surveill. 2004;9(12):6-8. PMID:15677856

11. Ho EL, Lukehart SA. Syphilis: using modern approaches to understand an old disease. J Clin Invest. 2011;121(12):4584-92. http://dx.doi.org/10.1172/JCI57173 PMID:22133883

12. Young H. Syphilis. Serology. Dermatol Clin. 1998;16(4):6918. http://dx.doi.org/10.1016/S0733-8635(05)70034-6 PMID:9891668

13. Grassly NC, Fraser C, Garnett GP. Host immunity and synchronized epidemics of syphilis across the United States. Nature. 2005;433(7024):417-21. http://dx.doi.org/10.1038/ nature03072 PMID:15674292

14. Morgan CA, Lukehart SA, Van Voorhis WC. Protection against syphilis correlates with specificity of antibodies to the variable regions of Treponema pallidum repeat protein K. Infect Immun. 
2003;71(10):5605-12. http://dx.doi.org/10.1128/IAI.71.10.56055612.2003 PMID:14500480

15. Ivens $D$, Patel $M$. Incidence and presentation of early syphilis diagnosed in HIV-positive gay men attending a central London outpatients' department. Int J STD AIDS. 2005;16(3):201-2. http://dx.doi.org/10.1258/0956462053420202 PMID:15829019

16. Winston A, Hawkins D, Mandalia S, Boag F, Azadian B, Asboe D. Is increased surveillance for asymptomatic syphilis in an HIV outpatient department worthwhile? Sex Transm Infect. 2003;79(3):257-9. http://dx.doi.org/10.1136/sti.79.3.257 PMID:12794218

17. Tramont EC. Treponema pallidum. In: Mandell GL, Bennett JE, Dolin R, editors. Principles and practice of infectious diseases. 6th ed. New York, NY: Churchill Livingstone Inc.; 2005. p. 2474-90.

18. Seña AC, White BL, Sparling PF. Novel Treponema pallidum serologic tests: a paradigm shift in syphilis screening for the 21st century. Clin Infect Dis. 2010;51(6):700-8. http://dx.doi. org/10.1086/655832 PMID:20687840

19. Centers for Disease Control and Prevention. Case definitions for infectious conditions under public health surveillance. MMWR Recomm Rep. 1997;46(RR-10):1-55. PMID:9148133

20. Chesney AM, Kemp JE. Studies in experimental syphilis: VI. On variations in the response of treated rabbits to reinoculation; and on cryptogenetic reinfection with syphilis. J Exp Med. 1926;44(5):589-606. http://dx.doi.org/10.1084/jem.44.5.589 PMID:19869209

21. Bremer V, Marcus U, Hamouda O. Syphilis on the rise again in Germany--results from surveillance data for 2011. Euro Surveill. 2012;17(29):20222. PMID:22835467

22. Velicko I, Unemo M. Recent trends in gonorrhoea and syphilis epidemiology in Sweden: 2007 to 2011. Euro Surveill. 2012;17(29):20223. PMID:22835468

23. European Centre for Disease Prevention and Control (ECDC). Sexually transmitted infections in Europe 2012. Stockholm: ECDC; 2014. Available from: http://www.ecdc.europa.eu/en/ publications/Publications/sexually-transmitted-infectionseurope-surveillance-report-2012.pdf

24. Epstein NN. Reinfection in syphilis. Calif Med. 1948;68(5):3747. PMID:18731447

25. Schoch AG, Alexander LJ. Reinfection and relapse after treatment of early syphilis with penicillin; analysis of 137 cases of infectious failure in a total series of 1,105 cases. Arch Derm Syphilol. 1949;60(5, Pt. 1):):690-700. http://dx.doi. org/10.1001/archderm.1949.01530050052004 PMID:15390444

26. Katz KA, Lee MA, Gray T, Marcus JL, Pierce EF. Repeat syphilis among men who have sex with men--San Diego County, 20042009. Sex Transm Dis. 2011;38(4):349-52. PMID:21099734

27. Brewer TH, Peterman TA, Newman DR, Schmitt K. Reinfections during the Florida syphilis epidemic, 2000-2008. Sex Transm Dis. 2011;38(1):12-7. http://dx.doi.org/10.1097/ OLQ.ob013e3181e9afc7 PMID:20739912

28. Hicks C. Diagnostic testing in syphilis. UpToDate. 2013. [Accessed 10 Jun 2013]. Available from: http://www.uptodate. com/contents/diagnostic-testing-for-syphilis

29. Hook EW 3̧rd, Martin DH, Stephens J, Smith BS, Smith K. A randomized, comparative pilot study of azithromycin versus benzathine penicillin $\mathrm{G}$ for treatment of early syphilis. Sex Transm Dis. 2002;29(8):486-90. http://dx.doi. org/10.1097/00007435-200208000-00010 PMID:12172535

30. Knaute DF, Graf N, Lautenschlager S, Weber R, Bosshard PP. Serological response to treatment of syphilis according to disease stage and HIV status. Clin Infect Dis. 2012;55(12):161522. http://dx.doi.org/10.1093/cid/cis757 PMID:22955437

31. McMillan A, Young H, Ogilvie MM, Scott GR, editors. Clinical practice in sexually transmissible infections: London WB Saunders/Elsevier Science; 2002. 